\title{
Medication non-adherence in inflammatory bowel diseases is associated with disability
}

\author{
Jonathan Perry ${ }^{1}$, Andy Chen ${ }^{2}$, Viraj Kariyawasam ${ }^{3}$, Glen Collins ${ }^{3}$, Chee Choong ${ }^{3}$, Wei Ling Teh ${ }^{3}$, \\ Nikola Mitrev ${ }^{1}$, Friedbert Kohler ${ }^{2}$, Rupert Wing Loong Leong ${ }^{1,2,3}$ \\ ${ }^{1}$ Sydney Medical School, University of Sydney, Sydney, ${ }^{2}$ Faculty of Medicine, University of New South Wales, Sydney, ${ }^{3}$ Gastroenterology and Liver \\ Services, Concord Hospital, Sydney, Australia
}

Background/Aims: Medication non-adherence is common in inflammatory bowel diseases (IBD). The short-term consequences of non-adherence include increased disease relapse but the long-term impact upon patients in terms of daily functional impairment are less well characterized. Identifying negative outcomes, such as disability, may encourage adherence. Methods: Consecutive ambulatory IBD subjects completed the Medication Adherence Rating Scale (MARS; non-adherence defined as $\leq 16$ ), Inflammatory Bowel Diseases Disability Index (IBD-DI; disability: <3.5) and Beliefs about Medicines Questionnaire (high necessity/concerns: $\geq 16$ ). The primary outcome was the association between medication non-adherence and disability. Secondary outcomes were the predictors of these outcomes. Results: A total of 173 subjects on IBD maintenance medications were recruited (98 Crohn's disease, 75 ulcerative colitis: median IBD-DI, -5.0 ; interquartile range [IQR], -14.0 to 4.0 and median MARS, 19.0; IQR, 18 to 20) of whom $24 \%$ were non-adherent. Disability correlated significantly with medication nonadherence $(r=0.38, P<0.0001)$. Median IBD-DI for non-adherers was significantly lower than adherers $(-16.0$ vs. $-2.0, P<0.0001)$. Predictors of disability included female sex $(P=0.002)$, previous hospitalization $(P=0.023)$, management in a referral hospital clinic $(P=0.008)$ and medication concerns $(P<0.0001)$. Non-adherence was independently associated with difficulty managing bowel movements (odds ratio [OR], 3.71; 95\% confidence interval [CI], 1.50-9.16, $P=0.005)$, rectal bleeding (OR, 2.69; 95\% CI, 1.14-6.36; $P=0.024)$ and arthralgia/arthritis (OR, 2.56; 95\% CI, 1.11-5.92; $P=0.028)$. Conclusions: Medication non-adherence was associated with significantly increased disability in IBD. Female gender, higher disease severity and medication concerns were additional predictors of disability. (Intest Res 2018;16:571-578)

Key Words: Crohn disease; Colitis, ulcerative; Inflammatory bowel diseases; Compliance; Drug

\section{INTRODUCTION}

Inflammatory bowel diseases (IBD) are chronic immunemediated inflammatory conditions encompassing UC and CD. ${ }^{1}$ The prevalence of these relapsing and remitting disorders has increased over time and they continue to be associated with significant morbidity. ${ }^{1,2}$ Persistently active disease is associated with debilitating gastrointestinal symptoms, extraintestinal manifestations, the development of complica-

Received March 7, 2018. Revised June 11, 2018. Accepted June 18, 2018. Correspondence to Rupert Wing Loong Leong, Gastroenterology and Liver Services, Concord Hospital, Hospital Rd, Sydney 2139, Australia. Tel: +61-29767-6111, Fax: +61-2-9767-6767, E-mail: rupertleong@outlook.com tions and bowel resection. ${ }^{3,4}$ IBD has been associated with a reduced quality of life. ${ }^{5}$ Quality of life, however, is a subjective measure of coping strategies. ${ }^{6}$ Disability is increasingly recognized as a more objective means of quantifying an illnesses' impact on day-to-day living, and is defined by the World Health Organization (WHO) as "any restriction or lack of ability to perform an activity in the manner or within the range considered normal. ${ }^{77}$ The Inflammatory Bowel Diseases Disability Index (IBD-DI) is a tool that measures the extent of functional impairment and disease burden in the IBD population. ${ }^{8}$ The IBD-DI is based upon the WHO's ICF (International Classification of Functioning, Disability and Health) core set which provides a framework for evaluating

\footnotetext{
๑ Copyright 2018. Korean Association for the Study of Intestinal Diseases. All rights reserved.

This is an Open Access article distributed under the terms of the Creative Commons Attribution Non-Commercial License (http://creativecommons.org/licenses/by-nc/4.0)

which permits unrestricted non-commercial use, distribution, and reproduction in any medium, provided the original work is properly cited.
} 
disability in terms of the interaction between the patients underlying illness and contextual environmental factors. ${ }^{8,9}$ The IBD-DI has been validated and correlated with disease activity and quality of life in both CD and UC. ${ }^{10}$ It also demonstrated real-life validity against decreased work productivity in IBD subjects from a referral clinic and increased direct and indirect healthcare cost in a population-based postoperative IBD cohort. ${ }^{10,11}$

Medication non-adherence occurs in up to $45 \%$ of IBD patients and is associated with increased symptoms and relapse rates. ${ }^{12}$ Non-adherence can be further categorized as intentional (deliberately stopping prescribed medications or altering their prescribed regime) and non-intentional (such as accidentally forgetting to take medications). The type of non-adherence may have implications for clinical outcomes and may have unique drivers. Unfortunately there has been no consistent predictor of non-adherence although some studies have identified younger age, full-time employment, shorter duration of disease, treatment type, psychological factors such as a strong emotional response and loss of response to therapy as possible risk factors. ${ }^{12-15}$ Non-adherence in IBD has been shown to be difficult for physicians to identify. ${ }^{16}$ To date there has been no simple and durable intervention that improves adherence rates. ${ }^{12}$

The impact of non-adherence includes increased risk of IBD relapse, increased disease activity and increased healthcare costs. ${ }^{17,18}$ The impact of non-adherence upon the day-to-day functioning of patients is less well characterized. Cumulative flares might result in cumulative intestinal complications, need for treatment escalation towards more potent medications and surgery. In the absence of a proven strategy that improves medication adherence, identifying the negative consequences of non-adherence might help convince patients to adhere to medications in order to avoid disability. Behavioral change might be expected from understanding the disabling consequences of recurrent flares. The primary aim of the study, therefore, was to identify the relationship between non-adherence and functional outcomes in IBD. Demographic, clinical and attitudinal factors were also assessed to determine predictors of adherence or disability.

\section{METHODS}

This was a cross-sectional non-interventional study to explore the association between medication non-adherence and disability. Disability is a concept that patients can relate to, being the result of many chronic diseases. The second- ary outcome measures were the predictors of disability and medication non-adherence as well as the impact of medication acceptance on adherence.

\section{Subjects}

Consecutive ambulatory patients aged $\geq 16$ years with a definitive diagnosis of CD or UC and $\geq 6$ months of follow-up were prospectively recruited. Patients recruited were within the catchment population of the Sydney IBD Cohort, which has a well-characterized population. ${ }^{19}$ Australia has a free universal healthcare system allowing subjects to be recruited from the full spectrum of socioeconomics. Inclusion criteria were being prescribed IBD maintenance medications. Exclusion criteria included insufficient English literacy and active psychological diagnoses such as mood disorders that impair day-to-day functioning.

Clinical and demographic information was obtained through self-report confirmed by their medical records. The data obtained included: demographics, IBD phenotype, duration of disease, recruitment from a dedicated IBD referral clinic or from private practice, membership of the patient support organization Crohn's and Colitis Australia (CCA), previous hospitalization or surgery related to IBD and the respondents' current medication regimen.

\section{Procedures}

Participant completed 3 questionnaires: IBD-DI, Medication Adherence Rating Scale (MARS), and the Beliefs about Medicines Questionnaire (BMQ) administered by an independent trained researcher separate to the medical consultation to reduce response bias.

\section{IBD Disability Index}

The IBD-DI is a validated questionnaire that measures disability in CD and UC. It has been validated for construct, discriminant ability, sensitivity to change and responsiveness. ${ }^{10}$ The IBD-DI comprises of 28 questions examining functional capabilities of patients with respect to the ICF core set and is significantly associated with disease activity and treatment received ${ }^{8,9}$ The tool is divided into 5 domains of overall health, body function, body structures, activity participation and environmental factors. The extent of patients limitations and disability are examined with respect to areas such as sleeping, mood, abdominal pain, bowel frequency, regulating defecation, participation in social events and work or school and exacerbating effects of medication, food, family and healthcare professionals. As a part of this study, the original questionnaire was reworded from a Flesch- 
Kincaid grade level 9 to grade level 6, an improvement recommended by previous studies, where subjects found the questionnaire would be improved with language simplification. A composite score ${ }^{10}$ represents the patients' extent of functional impairment or disability that ranges from 22 to -80. A more negative score correlates with greater disability. The IBD-DI demonstrates construct validity against the Inflammatory Bowel Disease Questionnaire (IBDQ), the disease activity indices CDAI for CD and the Mayo score in UC, predicts workforce participation and responsiveness. ${ }^{10}$

\section{Medication Adherence Rating Scale}

The MARS is a validated tool that has previously been utilized to evaluate adherence in IBD. ${ }^{20,21}$ Four statements relating to adherence are each scored on a 5-point Likert scale. The use of a scale avoids dichotomous "yes" or "no" answers that might result in response bias. Response bias is expected for any study of adherence where subjects exaggerate with medically-responsible answers. The MARS score ranges from 4 to 20 where a score of $\leq 16$ was defined as medication non-adherence. MARS is self-administered, easy to understand and completed confidentially in isolation remote to the consultation. The prescribing physician is blinded to the score to encourage patient honesty. MARS has been tested in IBD and correlates with markers of medication adherence such as serum drug levels and script dispensing data. ${ }^{21,22}$

\section{Beliefs about Medicines Questionnaire}

The BMQ is a validated survey tool that examines patient attitudes towards taking medications. ${ }^{14,21,23}$ It is based upon the beliefs about medications framework and consists of 10 items divided into statements exploring perceptions of the necessity for maintenance medications and the level of concern around their potential adverse effects. Respondents indicate their concordance with these statements on a 5-point Likert scale. A score is calculated for each of the domains of "medication concern" and "medication necessity." Scores range from 5 to 25 where respectively a higher score indicates a greater extent of concern regarding taking medications or a higher level of perceived necessity to take therapy. A score of $\geq 16$ in these 2 domains was defined as either "high concerns" or "high necessity" respectively. "Medication acceptance" was defined as perception of medications as being "high necessity" and "low concerns," which is considered to be the ideal attitude towards adherence to maintenance medications.

\section{Sample Size Calculation}

Sample size calculation was based on a quarter of IBD subjects meeting the threshold of disability from a previous study ${ }^{10}$ and a clinically meaningful doubling of disability resulting from medication non-adherence. Another study had identified the prevalence of medication-adherers to be 3 -fold higher than medication non-adherers in IBD. ${ }^{21}$ Based on a 2-sample proportional analysis for the trial to have $80 \%$ power on a 2-sided alpha level of $0.05,168$ consecutive subjects would be need to be recruited with a ratio of 3 medication adherers for every 1 medication non-adherer to detect a doubling of disability.

\section{Statistical Analysis}

Statistical analysis was used to evaluate associations between the MARS, BMQ and IBD-DI questionnaires and to determine the predictive clinical or demographic factors. Continuous variables were analyzed as non-parametric data using Spearman correlation, Mann-Whitney $U$-test for unpaired- and Kruskal-Wallis test for 3-continuous variables. Categorical variables were compared with chi-square or Fisher exact tests. Multivariate binomial logistic regression was utilized to determine the specific disability index outcomes that were independently associated with non-adherence. Multivariate linear regression modeling was used in analyzing adherence, beliefs about medicines and disability questionnaire scores. Analysis was performed using IBM SPSS version 22.0 (IBM Corp, Armonk, IL, USA). A $P$-value $<0.05$ was deemed statistically significant.

\section{Ethical Consideration}

Informed consent was obtained for all subjects involved in this study. The study was approved by the Concord Hospital and South Western Sydney Local Health District Human Research and Ethics Committees (Reference numbers: LNR/14/CRGH/69; LNRSSA/15/LPOOL/313) in accordance with to the ethical guidelines of the 1975 Declaration of Helsinki (6th revision, 2008).

\section{RESULTS}

\section{Study Population}

In total 173 respondents (98 CD, $75 \mathrm{UC}$; median age 40.0, $51 \%$ females) were prospectively recruited for this study between March 2014 and September 2015. Through consecutive recruitment, specialist referral IBD clinic patients made up $64 \%$ of the cohort while the remainder were recruited from private gastroenterology practices. The duration of 
illness was not significantly different between care setting $(P=0.82)$. However, referral clinic patients were more likely to have CD than those seen in private practice ( $71 \%$ vs. $55 \%$, $P=0.02)$. Maintenance therapy with an immunomodulator was more common in CD versus UC $(72 \%$ vs. $32 \% P<0.01)$. Anti-tumor necrosis factor therapy utilization was more prevalent amongst CD patients than those with UC (37\% vs. $1 \% P<0.01)$. Mesalazine use in $\mathrm{CD}$ was $36 \%$ compared to $89 \%$ amongst the UC cohort $(P<0.01)$. Previous IBD-related hospitalization and surgery respectively was reported in $47 \%$ and $32 \%$ of CD patients compared to $28 \%$ and $5 \%$ of UC patients $(P<0.01)$. Our population data set was not-normally distributed and was therefore analyzed using non-parametric tests.

\section{Non-Adherence and Disability}

Of all subjects recruited, $24 \%(\mathrm{n}=42)$ were non-adherent with their IBD maintenance medications. Non-adherers had similar baseline characteristics to adherers (Table 1). Medication non-adherence correlated significantly with worse disability (correlation of MARS adherence score vs. IBD-DI, $r=0.38$, $P<0.0001$; non-adherers median IBD-DI score -16.0 [IQR, -26.0 to -6.0 ] vs. adherers -2.0 [IQR, -9.0 to 5.0$], P<0.0001$ ) (Fig. 1). Non-adherence correlated significantly with perceived non-necessity of medications $(r=0.22, P=0.004)$ and with concern about medical treatment $(r=0.32, P<0.0001)$. Non-adherent patients demonstrated significant associations with disability in the composite IBD-DI and in all of its 5 domains of overall health, body function, body structures, activity participation and environmental factors (Table 2).

\section{Factors Affecting Disability}

Disability was not associated with IBD disease phenotype $(P=0.41)$ or IBD duration greater than 5 years $(P=0.39)$. However, disability was significantly association with previous hospitalization $(P=0.010)$, previous surgery $(P=0.035)$, the use of corticosteroid therapy $(P=0.007)$, management through a referral clinic $(P<0.0001)$ and female sex $(P=0.002)$

Table 1. Demographics of Respondents and Outcome Measures According to Medication Adherence

\begin{tabular}{|c|c|c|c|}
\hline & $\begin{array}{l}\text { Adherent subjects } \\
\qquad(n=131)\end{array}$ & $\begin{array}{l}\text { Non-adherent subjects } \\
\qquad(n=42)^{\mathrm{a}}\end{array}$ & $P$-value \\
\hline \multicolumn{4}{|l|}{ Demographic } \\
\hline Female sex & 65 (49.6) & $24(57.1)$ & 0.400 \\
\hline$C D$ & $79(60.3)$ & $19(45.2)$ & 0.090 \\
\hline Married/relationship & $70(53.4)$ & $24(57.1)$ & 0.680 \\
\hline Crohn's and Colitis Australia membership & $16(12.2)$ & $8(19.0)$ & 0.260 \\
\hline Previous IBD related hospitalization & $52(39.7)$ & $15(35.7)$ & 0.640 \\
\hline Previous IBD related surgery & $26(19.8)$ & $9(21.4)$ & 0.820 \\
\hline Corticosteroid & $31(23.7)$ & $9(21.4)$ & 0.760 \\
\hline Anti-tumor necrosis factor $\alpha$ agent & $32(24.4)$ & $5(11.9)$ & 0.085 \\
\hline \multicolumn{4}{|l|}{ Key questionnaire outcome measures } \\
\hline High medication concerns & $58(44.3)$ & $31(73.8)$ & 0.001 \\
\hline High medication necessity & $121(92.4)$ & $33(78.6)$ & 0.013 \\
\hline Medication acceptance & $66(50.4)$ & $8(19.0)$ & $<0.001$ \\
\hline Disability (IBD-DI score <3.5) & 94 (71.8) & $38(90.5)$ & 0.013 \\
\hline IBD-DI (score) & $-2(-9$ to 5$)$ & $-16(-26$ to -6$)$ & $<0.001$ \\
\hline
\end{tabular}

Value are presented as median (interqutile range) or number (\%).

${ }^{a}$ Non-adherence defined by Medication Adherence Reporting Scale score $\leq 16$, range 4-20.

IBD-DI, Inflammatory Bowel Diseases Disability Index. 
(Table 3). Independent predictors of poorer functional outcomes on IBD-DI linear regression were high medication concern $(P<0.0001)$, management through a referral clinic $(P=0.008)$, previous hospitalization $(P=0.023)$, and female $\operatorname{sex}(P=0.008)$.

\section{Clinical Features Associated with Non-Adherence}

On multivariate binary logistic regression 3 disease features were identified as being independently associated with medication non-adherence. These are: difficulty managing bowel movements (OR, 3.71; 95\% CI, 1.50-9.16; $P=0.005$ ), the presence of rectal bleeding $(\mathrm{OR}, 2.69 ; 95 \% \mathrm{CI}, 1.14-6.36$; $P=0.024)$ and the presence of arthralgia or arthritis (OR, 2.56; 95\% CI, 1.11-5.92; $P=0.028$ ) (Table 4). Anxiety trended towards significance within the model (OR, 2.83; 95\% CI, 0.91-8.81; $P=0.073$ ) while other functional outcomes exam-

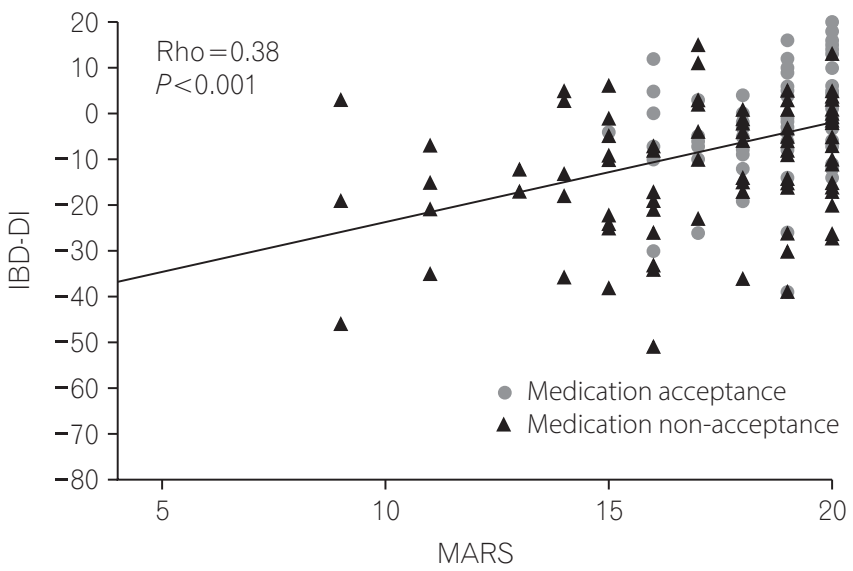

Fig. 1. Correlation between Medication Adherence Rating Scale (MARS) and the Inflammatory Bowel Diseases Disability Index (IBD-DI) according to acceptance of medications. Medication acceptance is defined as a subject's perception of high medication necessity and low concerns according to their Beliefs about Medicines Questionnaire. ined in the IBD-DI demonstrated no significant independent association with adherence.

Table 3. Factors Associated with Disability as Measured by IBD-DI

\begin{tabular}{|c|c|c|}
\hline & $\begin{array}{l}\text { Median difference } \\
\text { in IBD-DI score }\end{array}$ & $P$-value \\
\hline \multicolumn{3}{|l|}{ Socio-demographic } \\
\hline Age & $-0.032^{\mathrm{a}}$ & 0.670 \\
\hline Female sex & -7.0 & 0.002 \\
\hline Married/relationship & -1.0 & 0.700 \\
\hline $\begin{array}{l}\text { Crohn's \& Colitis Australia } \\
\text { membership }\end{array}$ & -1.5 & 0.730 \\
\hline Tertiary IBD clinic management & -5.5 & $<0.001$ \\
\hline \multicolumn{3}{|l|}{ Disease characteristics } \\
\hline Diagnosis greater than 5 years ago & -3.0 & 0.390 \\
\hline UC diagnosis & 1.0 & 0.405 \\
\hline Previous IBD related hospitalization & -4.5 & 0.010 \\
\hline Previous surgery & -4.0 & 0.035 \\
\hline \multicolumn{3}{|l|}{ Treatment modalities } \\
\hline 5-ASA & 0.5 & 0.500 \\
\hline Immunomodulator & -1.0 & 0.450 \\
\hline Steroids & -5.5 & 0.007 \\
\hline Biologicals & 2.0 & 0.770 \\
\hline \multicolumn{3}{|l|}{ Beliefs about medications } \\
\hline High concerns & -5.0 & $<0.001$ \\
\hline Low necessity & 4.0 & 0.800 \\
\hline Lack of acceptance & -7.5 & $<0.001$ \\
\hline \multicolumn{3}{|l|}{ Medication related behavior } \\
\hline Non-adherence & -14.0 & $<0.001$ \\
\hline
\end{tabular}

Table 2. IBD-DI Scores According to the International Classification of Functioning Domains Comparing Adherent versus Non-Adherent Subjects

\begin{tabular}{|c|c|c|c|}
\hline \multirow[b]{2}{*}{ Domain } & \multicolumn{2}{|c|}{ IBD-DI score } & \multirow[b]{2}{*}{$P$-value } \\
\hline & $\begin{array}{l}\text { Adherent subject } \\
\qquad(n=131)\end{array}$ & $\begin{array}{l}\text { Non-adherent subjects } \\
\qquad(\mathrm{n}=42)\end{array}$ & \\
\hline Overall health & $-1(-1.5$ to 1.5$)$ & $-2(-3$ to -3$)$ & $<0.001$ \\
\hline Body function & $-6(-10$ to -2$)$ & $-11(-16.6$ to -5.4$)$ & $<0.001$ \\
\hline Body structure & 2 (1 to 3$)$ & $0(-1.3$ to 1.3$)$ & $<0.001$ \\
\hline Activity participation & $-2(-4.5$ to 0.5$)$ & $-6(-9.5$ to -2.5$)$ & $<0.001$ \\
\hline Environmental factors & 6 (2.5 to 9.5$)$ & 3 (0 to 6$)$ & $<0.001$ \\
\hline Total score & $-2(-9.0$ to 5.0$)$ & $-16(-26.0$ to -6.0$)$ & $<0.001$ \\
\hline
\end{tabular}

Values are presented as median (interquartile range).

IBD-DI, Inflammatory Bowel Diseases Disability Index. 
Table 4. Logistic Regression Model of Disease Features Associated with Medication Non-Adherence

\begin{tabular}{lccc}
\hline Clinical outcome & OR $(95 \% \mathrm{Cl})$ & $\beta$ Coefficient & $P$-value \\
\hline Fatigue & $2.60(0.71-9.46)$ & 0.96 & 0.150 \\
Low mood & $0.53(0.18-1.55)$ & -0.63 & 0.250 \\
Anxiety & $2.83(0.91-8.81)$ & 1.04 & 0.073 \\
Abdominal pain & $0.46(0.17-1.23)$ & -0.78 & 0.120 \\
$\begin{array}{l}\text { Difficulty managing } \\
\quad \text { defecation }\end{array}$ & $3.71(1.50-9.16)$ & 1.31 & 0.005 \\
$\begin{array}{l}\text { Presence of liquid } \\
\quad \text { stools }\end{array}$ & $0.58(0.23-1.47)$ & -0.54 & 0.250 \\
$\quad$ Weight loss & $1.74(0.67-4.51)$ & 0.55 & 0.260 \\
Rectal bleeding & $2.69(1.14-6.36)$ & 0.99 & 0.024 \\
Arthralgia/arthritis & $2.56(1.11-5.92)$ & 0.94 & 0.028 \\
\hline
\end{tabular}

\section{DISCUSSION}

Medication non-adherence in IBD is associated with increased risk of relapse and healthcare utilization. ${ }^{12,17,24,25}$ These intangible concepts, however, might be insufficient to motivate patients in improving their adherence. Disability is a sustained, recognizable, relevant and self-identifiable concept that might be a greater motivational force to improve adherence. This study demonstrated a significant association between medication non-adherence and disability. Medication non-adherence was significantly associated with impaired activity pariticipation and impairment of all IBDDI domains. Disability was also significantly associated with symptoms of flares, requirement for corticosteroids, IBDrelated hospitalization and surgery. The avoidance of disability, therefore, becomes a shared goal between the physician and patient. As such, the translatable benefits of this study might be in shaping a conversation that motivates patients to improve their adherence in order to prevent or reverse disability. The quantification of disability through the IBD-DI may also have utility at the level of healthcare organisations as significant outcome measure or quality indicator. ${ }^{26}$

Three specific symptoms of IBD are significantly independently associated with medication non-adherence. Arthralgia and rectal bleeding are the symptoms associated with non-adherence with greatest frequency, while difficulty managing defecation has the strongest association within the model. All of these factors are prevalent in active IBD and have been associated with impaired quality of life and impaired social engagement. ${ }^{27-30}$ Presentation with these symptoms, therefore, might alert the physician of possible medication non-adherence.
Apart from non-adherence, disability was also independently associated with previous hospitalization, management in an IBD referral center, having high concerns over medications and female sex. Previous hospitalization and management in a referral center represent greater disease severity. Disability in IBD has also been found to be associated with female sex. ${ }^{10,31}$ Symptoms of anxiety and depression that might result in avoidance of using medications are also associated with disability. ${ }^{32}$ Managing medication nonadherence, therefore, might require a targeted approach in identifying high-risk subjects, those with severe disease and treatment of psychological comorbidities. Promising preliminary results have been seen with a pharmacist-led personalized educational intervention. ${ }^{33}$

This study has several limitations. Firstly, although there was a strong association between non-adherence and disability, causality cannot be confirmed. Although the logic is that non-adherence results in cumulative disease flares that result in chronic disease activity and/or irreversible intestinal damage, the alternative explanation that cumulative disability leads to medication non-adherence is possible. This was an observational cross-sectional study which inherently is limited in its power to detect causality and is able to only establish associations. Ultimately a randomized controlled longitudinal study is required to demonstrate decreased disability through improved adherence. Although there is a clear linear correlation between disability and adherence score the strength of this correlation is reduced somewhat due to a wide range of IBD-DI scores for a given range of the MARS. Secondly, adherence level may fluctuate over time. However, we recently demonstrated that baseline adherence or any improvement in adherence through counseling is consistent even when followed up for 2 years. ${ }^{33}$ Medication adherence is, therefore, likely a feature of the subjects' pervasive beliefs and perception. Thirdly, this was not a population-based study. However, the subjects were recruited from both referral and private practice settings to provide a range of IBD disease characteristics. Our data are consistent with a French population-based study ${ }^{31}$ that also demonstrated greater disability levels in females and those with arthralgia/ arthritis, and the lack of association between disability with IBD phenotype and duration of IBD. It is likely, therefore, that this study can be generalizable. Fourthly adherence and disability data relied on patient self-reporting. However, the MARS is a commonly-used tool that reflects real-world multiple medication usage rather than limiting to either single drug class or drugs that have bioassays. Unlike the Morisky et al. ${ }^{34}$ MMAS-8 tool MARS uses Likert scales in eliciting sub- 
ject responses. By avoiding dichotomous "yes" or "no" replies, subjects are less likely to exaggerate their answers towards those that are more "medically responsible." Similarly the IBD-DI has been validated against work productivity and quality of life specific to IBD. ${ }^{10}$ Beliefs about Medications Questionnaire has been also previously evaluated in IBD to provide certain degrees of external validation. ${ }^{14,20}$ In addition we have not examined the impact of adherence to non-pharmacological treatments which may also impact significantly on disability. If a larger sample size had allowed for stratification by phenotype this may have been informative, however overall there was no association between phenotype and adherence/disability. By design patients in this study did not complete survey tools to assess diseases activity such as the CDAI or pMayo scores.

The IBD-DI has previously been validated ${ }^{10}$ against these disease activity measures and its use as an independent outcome measure as in this study enhances its utility, additionally it was predicted that patients would experience a degree of survey fatigue if the set of administered tools was expanded any further. Laboratory or endoscopic data was not used to evaluate the results in this study as our aim was to assess functional impairment/disability broadly rather than disease activity or detect episodes of relapse. Strengths of the study include a recruitment sample size that was achieved, the use of validated tools for IBD and not limiting the subjects to tertiary hospital subjects.

In conclusion, poor medication adherence to IBD maintenance therapy is strongly associated with an increased level of disability. Uncontrolled active IBD through medication non-adherence might result in flares, need for hospitalization and eventual treatment escalation. Predictors of both non-adherence and disability included the presence of anxiety symptoms while female sex and higher disease severities as evident by prior hospitalization predicted increased disability. Patients with these adverse features require specific management in order to reduce their risk of long-term disability. The implementation of programs targeted towards addressing medication concerns and knowledge deficits in at risk patients may lead to the reversal of non-adherence in order to reduce long-term functional impairment.

\section{FINANCIAL SUPPORT}

The authors received no financial support for the research, authorship, and/or publication of this article.

\section{CONFLICT OF INTEREST}

All authors declare that they have no conflicts of interest with respect to this work. Dr. Rupert Wing Loong Leong reports advisory board membership from Takeda, Janssen, AbbVie, MSD, Pfizer, Ferring and Aspen unrelated to this research.

\section{AUTHOR CONTRIBUTION}

Conceptualization: JP, AC, RL. Methodology: JP, AC, RL, CC, GC, WLT. Formal analysis: JP, AC, RL, FK, NM, WLT, VK. Project administration: RL, GC, WLT, CC Visualization: JP, AC. Writing - original draft: JP, AC RL, FK, GC. Writing - review and editing: JP, RL, FK, VK, CC, WT, NM, GC. Approval of final manuscript: all authors.

\section{ACKNOWLEDGEMENTS}

The authors acknowledge Wei Xuan, Ingham Institute of Applied Medical Research, UNSW, Sydney, New South Wales, Australia, for statistical support.

\section{REFERENCES}

1. Ananthakrishnan AN. Epidemiology and risk factors for IBD. Nat Rev Gastroenterol Hepatol 2015;12:205-217.

2. Ko Y, Butcher R, Leong RW. Epidemiological studies of migration and environmental risk factors in the inflammatory bowel diseases. World J Gastroenterol 2014;20:1238-1247.

3. Vatn MH. Natural history and complications of IBD. Curr Gastroenterol Rep 2009;11:481-487.

4. Ott C, Schölmerich J. Extraintestinal manifestations and complications in IBD. Nat Rev Gastroenterol Hepatol 2013;10:585595.

5. Lix LM, Graff LA, Walker JR, et al. Longitudinal study of quality of life and psychological functioning for active, fluctuating, and inactive disease patterns in inflammatory bowel disease. Inflamm Bowel Dis 2008;14:1575-1584.

6. Peyrin-Biroulet L. What is the patient's perspective: how important are patient-reported outcomes, quality of life and disability? Dig Dis 2010;28:463-471.

7. World Health Organization. Document A29/INFDOCI/1. Geneva: WHO, 1976.

8. Peyrin-Biroulet L, Cieza A, Sandborn WJ, et al. Development of the first disability index for inflammatory bowel disease based on the international classification of functioning, disability and health. Gut 2012;61:241-247. 
9. Lo B, Prosberg MV, Gluud LL, et al. Systematic review and metaanalysis: assessment of factors affecting disability in inflammatory bowel disease and the reliability of the inflammatory bowel disease disability index. Aliment Pharmacol Ther 2018;47:6-15.

10. Leong RW, Huang T, Ko Y, et al. Prospective validation study of the International Classification of Functioning, Disability and Health score in Crohn's disease and ulcerative colitis. J Crohns Colitis 2014;8:1237-1245.

11. Lee Y, McCombie A, Gearry R, et al. Disability in restorative proctocolectomy recipients measured using the Inflammatory Bowel Disease Disability Index. J Crohns Colitis 2016;10:13781384.

12. Selinger CP, Robinson A, Leong RW. Clinical impact and drivers of non-adherence to maintenance medication for inflammatory bowel disease. Expert Opin Drug Saf 2011;10:863-870.

13. Jackson CA, Clatworthy J, Robinson A, Horne R. Factors associated with non-adherence to oral medication for inflammatory bowel disease: a systematic review. Am J Gastroenterol 2010;105:525-539.

14. Ediger JP, Walker JR, Graff L, et al. Predictors of medication adherence in inflammatory bowel disease. Am J Gastroenterol 2007;102:1417-1426.

15. van der Have M, Oldenburg B, Kaptein AA, et al. Non-adherence to anti-TNF therapy is associated with illness perceptions and clinical outcomes in outpatients with inflammatory bowel disease: results from a prospective multicentre study. J Crohns Colitis 2016;10:549-555.

16. Trindade AJ, Ehrlich A, Kornbluth A, Ullman TA. Are your patients taking their medicine? Validation of a new adherence scale in patients with inflammatory bowel disease and comparison with physician perception of adherence. Inflamm Bowel Dis 2011;17:599-604.

17. Higgins PD, Rubin DT, Kaulback K, Schoenfield PS, Kane SV. Systematic review: impact of non-adherence to 5-aminosalicylic acid products on the frequency and cost of ulcerative colitis flares. Aliment Pharmacol Ther 2009;29:247-257.

18. Chan W, Chen A, Tiao D, Selinger C, Leong R. Medication adherence in inflammatory bowel disease. Intest Res 2017;15:434445.

19. Selinger CP, Andrews JM, Titman A, et al. Long-term follow-up reveals low incidence of colorectal cancer, but frequent need for resection, among Australian patients with inflammatory bowel disease. Clin Gastroenterol Hepatol 2014;12:644-650.

20. Horne R, Parham R, Driscoll R, Robinson A. Patients' attitudes to medicines and adherence to maintenance treatment in inflammatory bowel disease. Inflamm Bowel Dis 2009;15:837844.
21. Selinger CP, Eaden J, Jones DB, et al. Modifiable factors associated with nonadherence to maintenance medication for inflammatory bowel disease. Inflamm Bowel Dis 2013;19:21992206 .

22. Fialko L, Garety PA, Kuipers E, et al. A large-scale validation study of the Medication Adherence Rating Scale (MARS). Schizophr Res 2008;100:53-59.

23. Horne R, Weinman J, Hankins M. The beliefs about medicines questionnaire: the development and evaluation of a new method for assessing the cognitive representation of medication. Psych Health 1999;14:1-24.

24. Kane S, Shaya F. Medication non-adherence is associated with increased medical health care costs. Dig Dis Sci 2008;53:10201024.

25. Sellin J. Disability in IBD: the devil is in the details. Inflamm Bowel Dis 2010;16:23-26.

26. Berry SK, Melmed GY. Quality indicators in inflammatory bowel disease. Intest Res 2018;16:43-47.

27. Brakenhoff LK, van der Heijde DM, Hommes DW. IBD and arthropathies: a practical approach to its diagnosis and management. Gut 2011;60:1426-1435.

28. Stange EF, Travis SP, Vermeire S, et al. European evidence-based consensus on the diagnosis and management of ulcerative colitis: definitions and diagnosis. J Crohns Colitis 2008;2:1-23.

29. Stange EF, Travis SP, Vermeire S, et al. European evidence based consensus on the diagnosis and management of Crohn's disease: definitions and diagnosis. Gut 2006;55 Suppl 1:i1-i15.

30. Norton C, Dibley LB, Bassett P. Faecal incontinence in inflammatory bowel disease: associations and effect on quality of life. J Crohns Colitis 2013;7:e302-e311. doi: 10.1016/j.crohns.2012.11.004.

31. Gower-Rousseau C, Sarter H, Savoye G, et al. Validation of the Inflammatory Bowel Disease Disability Index in a populationbased cohort. Gut 2017;66:588-596.

32. Chan W, Shim HH, Lim MS, et al. Symptoms of anxiety and depression are independently associated with inflammatory bowel disease-related disability. Dig Liver Dis 2017;49:13141319.

33. Tiao DK, Chan W, Jeganathan J, et al. Inflammatory bowel disease pharmacist adherence counseling improves medication adherence in Crohn's disease and ulcerative colitis. Inflamm Bowel Dis 2017;23:1257-1261.

34. Morisky DE, Ang A, Krousel-Wood M, Ward HJ. Predictive validity of a medication adherence measure in an outpatient setting. J Clin Hypertens (Greenwich) 2008;10:348-354. 